\title{
Caries Color, Extent, and Preoperative Pain as Predictors of Pulp Status in Primary Teeth
}

This article was published in the following Dove Press journal:

Clinical, Cosmetic and Investigational Dentistry

\author{
Faezeh Ghaderi' \\ Zahra Jowkar (iD) ${ }^{2}$ \\ Alireza Tadayon ${ }^{3}$ \\ 'Oral and Dental Disease Research \\ Center, Department of Pediatric \\ Dentistry, School of Dentistry, Shiraz \\ University of Medical Sciences, Shiraz, \\ Iran; ${ }^{2}$ Oral and Dental Disease Research \\ Center, Department of Operative \\ Dentistry, School of Dentistry, Shiraz \\ University of Medical Sciences, Shiraz, \\ Iran; ${ }^{3}$ Department of Operative \\ Dentistry, School of Dentistry, Shiraz \\ University of Medical Sciences, Shiraz, \\ Iran
}

Aim: This study aimed to assess the relationship between preoperative pain status, caries extent, and caries color and the ability to achieve hemostasis at the canal orifices during pulp therapy access in primary teeth.

Study Design: Eighty primary molar teeth with extremely deep caries extending to the pulp (based on bitewing radiography) were investigated in 66 children. The teeth with clear radiographic and clinical signs indicating the involvement of the radicular pulp were excluded. The history of preoperative pain status, the color of dental caries by visual inspection, and the extent of caries lesion measured in bitewing radiographs were recorded. After preparing the access cavities, the hemostasis achievement at the canal orifices was assessed and considered as the final factor in determining the type of treatment (pulpotomy or pulpectomy). The data were analyzed using Fisher's exact test, the independent $t$-test, and Cohen's unweighted kappa statistic. $\mathrm{P}<0.05$ was considered statistically significant.

Results: Hemostasis was achieved in $78.1 \%$ of the teeth with black dental caries, whereas in most of the teeth with light brown dental caries (64.3\%), hemostasis was not achieved $(\mathrm{p}=0.015)$. The extent of dental caries in the teeth needing pulpotomy was statistically significantly lower than that of those needing pulpectomy $(\mathrm{p}=0.024)$. Mesial side dental caries was more related to the irreversible pulpitis compared to the distal one $(p=0.01)$. In $78.1 \%$ of the teeth without pain, hemostasis was achieved, and in most of the teeth with nocturnal pain $(64.3 \%)$, hemostasis did not occur $(\mathrm{p}=0.034)$.

Conclusion: The caries color, caries extent, and preoperative pain have the potential to be used as clinical diagnostic tools to determine the pulpal status in primary teeth.

Keywords: caries color, caries extent, hemostasis, pain, pulp treatment

\section{Introduction}

Pulp diagnosis in pediatric dentistry is very critical in selecting the correct treatment strategy in primary teeth. ${ }^{1}$ This provides more information about the duration and cost of treatment as well as the pain experienced by the patients during treatment. Therefore, the practitioner can evaluate whether any pretreatment method for the reduction of pain perception would be needed. ${ }^{2,3}$ The histological examination can exactly determine the extent of inflammation or the presence of necrosis in the pulp tissue. Therefore, it has been considered as the most accurate way for the assessment of the pulp status. ${ }^{4}$ However, it is not clinically possible to determine the histological status of the pulp. Therefore, clinicians should rely more on the patient's history and the clinical and radiographic assessment of the signs and symptoms to determine the treatment options. ${ }^{5,6}$

Although there is controversy about whether the achievement of hemostasis after pulp exposure would provide an accurate assessment of pulp inflammation, ${ }^{7,8}$
Correspondence: Zahra Jowkar

Tel $+98-71-36263193$

Fax +98-7I-36280804

Email zahrajowkar66@gmail.com 
the final clinical indicator in the assessment of pulpal inflammation and its extension into the root canals in primary teeth is hemostasis during pulp therapy access in primary teeth. ${ }^{8}$

Reversible pulpitis is usually symptomless or has less intense and shorter-lasting episodes of pain compared with irreversible pulpitis. In contrast, the spontaneous and radiating pain that lingers after the removal of the stimulus tends to be indicative of irreversible pulpitis. ${ }^{9}$ However, irreversible pulpitis might sometimes be symptomless. ${ }^{9}$ Conversely, the absence of pain does not necessarily mean that the pulp is free from widespread inflammation. Moreover, pediatric patients generally have a very low pain threshold compared to adults. Thus, they cannot always describe subjective symptoms or sensibility to a stimulus. ${ }^{8}$

There are a few studies that have evaluated the correlation between the clinical properties of dental caries and pulp status. ${ }^{6,10}$ The level of caries activity within a lesion cannot be identified objectively. Therefore, clinicians use clinical judgments and subjective measures for caries activity assessment. ${ }^{11}$ At present, the color and hardness of carious lesions are two clinical criteria used for the diagnosis of the degree of carious progression and carious lesion activity. ${ }^{12,13}$ An active caries tends to be light yellow/beige, whereas slowly progressing or arrested caries lesions are usually darker. ${ }^{14}$

Another diagnostic tool for obtaining a provisional primary diagnosis of the pulp status is the radiographic examination which can indicate potential pulp necrosis and apical pathosis. ${ }^{9}$ Additionally, the depth of a carious lesion can be estimated with a bitewing radiography. ${ }^{15}$

Caries extent can be indicative of inflammatory changes within the pulp. A previous study showed that the teeth with a carious breakdown of more than half of the buccolingual intercuspal distance are more likely to have some inflammatory changes within the pulp horn region compared to the teeth with a carious breakdown of less than half of the buccolingual intercuspal distance. ${ }^{10}$

To the best of our knowledge, only one study has been conducted to determine whether the pulp status can be predicted in primary teeth before treatment which demonstrated the efficacy of using an electric pulp tester in assessing the pulp status in primary teeth. ${ }^{8}$ Therefore, this study aimed to assess the relationship between preoperative pain status, caries extent, and caries color and the ability to achieve hemostasis at the canal orifices during pulp therapy access in primary teeth.

\section{Materials and Methods}

The study protocol was approved by the Research and Ethics Committee of Shiraz University of Medical Sciences (IR.SUMS.DENTA1.REC.1398.27). The parents of all the participants in this study signed the informed consent after the nature, the possible discomforts, and the risks of the procedures were explained to them. Children with behavioral problems or systemic disorders were not included in this study.

\section{The Inclusion and Exclusion Criteria}

Patients with carious primary molar teeth in need of pulp therapy (pulpotomy or pulpectomy) were enrolled in this study. The inclusion criteria for the teeth used in this study were as follows: normal furcation, normal periapical bone structure, no root resorption, and no periodontal ligament widening evident on a periapical radiograph. The exclusion criteria in this study were as follows: teeth with mobility, periapical abscess, fistula, or buccal or lingual dental caries. Moreover, the teeth with buccal or lingual caries were excluded from the study due to the superimposition of buccal or lingual caries in the radiographs.

\section{The Study Protocol}

All the participants were 5-12 years old and had bitewings indicating a deep caries lesion needing pulp therapy. A pilot study was conducted with 10 teeth treated with pulpotomy and 10 teeth treated with pulpectomy to calculate the optimal sample size. The teeth which were treated in the pilot study were not considered as the main cases of the study. A sample size of 62 teeth was required based on the pilot study considering the power of 0.8 and $\alpha=0.05$. The sample size was increased to 80 teeth to compensate for the potential dropouts.

The extent of caries lesion (based on bitewing radiography), the history of pain status, and the color of dental caries were assessed and recorded.

\section{The Clinical Assessment of Dental Caries Color}

The color of dental caries was determined via visual inspection under the light of the unit by two independent observers. The caries colors were classified as black, dark brown, light brown, and yellow. ${ }^{14,16}$ The clinical observation of the dental caries color was compared with photographs illustrating the four typical classes of caries color. ${ }^{14}$ 
For the recorded color of caries, the interobserver correlation coefficient was 0.8 .

\section{Dental Caries Extension}

For dental caries extension, the mesiodistal width of the teeth and the greatest diameter of the caries lesion were measured based on bitewing radiography. Then, the largest diameter of the caries lesion was divided by the mesiodistal width of the teeth to obtain a ratio. This ratio was used as an index for the evaluation of the caries extent. Moreover, the tooth surfaces involved with dental caries (mesial, distal, and occlusal) were recorded. All the measurements for dental caries extension were done by a calibrated operator.

\section{Pain History}

The type of preoperative pain reported by the patient or parent which seemed to be related to the tooth needing treatment was recorded and classified into four categories (no pain, positive elicited pain, spontaneous pain, and nocturnal pain) based on the detailed history of pain. Provoked or elicited pain was registered if the patient reported pain when eating or drinking cold, hot, or sweet foods or beverages and during mastication in the area under study.

\section{The Clinical Procedures of the Treatment}

After the administration of anesthesia and rubber dam isolation of the selected tooth, a round diamond bur mounted on a high-speed handpiece was used with copious water supply to reach the dentinal carious lesions. Afterward, a carbide round bur in a slow-speed handpiece was used for the removal of the infected carious dentin. If the dental pulp was not exposed after complete caries removal, the tooth was restored and the patient was excluded from the study.

After drilling the ceiling of the pulp chamber in the exposed teeth using an excavator or a round carbide bur in a slow-speed handpiece without water, the coronal pulp tissue was removed using a spoon excavator and the coronal pulp chamber was washed with normal saline. A sterile saline-wetted cotton pellet was placed into the chamber with pressure for $5 \mathrm{~min}$. If hemostasis was achieved after the removal of the cotton pellet, the tooth was treated with pulpotomy by applying 15.5\% ferric sulfate (Astringedent; Ultradent, South Jordan, Utah,
USA) with sterilized cotton pellets over the remaining pulpal tissue at the canal orifice for 10-15 s. Then, the ferric sulfate was rinsed with sterilized saline water, zinc oxide eugenol paste was placed, and the cavity was restored with amalgam (Permite, SDI).

If hemostasis was not achieved within $5 \mathrm{~min}$ of pressure with the saline moistened cotton pellet, pulpectomy was carried out. In the pulpectomy cases, the radicular pulp tissue was removed with a barbed broach and the root canal was instrumented with Kerr files. Then, the root canal was irrigated with saline solution and dried and filled with zinc oxide-eugenol (ZOE) paste. Afterward, the tooth was restored with amalgam. The type of treatment and the achievement of hemostasis in each tooth were recorded.

\section{The Statistical Analysis}

The data were analyzed using SPSS software version 17 (SPSS Inc., Chicago, USA). P-values of less than 0.05 were considered statistically significant. Fisher's exact test was used to analyze the data of the preoperative pain status and the achievement of hemostasis and also to analyze the data of caries color and hemostasis achievement. An independent $t$-test was used to assess the associations between caries extent and hemostasis achievement. The chi-square test was used to analyze the data of the surfaces of the teeth which were involved by caries and the achievement of hemostasis. The intra-examiner reproducibility for determining the type of pulp treatment before starting the treatment and after access cavity preparation was calculated by Cohen's unweighted kappa statistic.

\section{Results}

In this study, 80 primary first and second molar teeth were investigated in 66 children (33 girls and 33 boys) with a mean age of $6.90 \pm 1.39$ years. The numbers of the first upper molar, first lower molar, second upper molar, and second lower molar teeth were 19 (23.8\%), 32 $(40 \%), 20(25 \%)$, and $9(11.3 \%)$, respectively. Pulpal exposure occurred after caries removal in all of the teeth.

Fisher's exact test was used to analyze the data of the preoperative pain status and the achievement of hemostasis $(p=0.034)$ (Table 1$)$. In most of the teeth without pain (25 out of 32 teeth, 78.1\%), hemostasis was achieved. Hemostasis did not occur in most of the teeth with nocturnal pain (9 out of 14 teeth, 64.3\%).

Fisher's exact test was also used to analyze the data of caries color and the achievement of hemostasis $(p=0.015)$ (Table 1). None of the teeth in the study had yellow dental 
Table I The Frequency of the Treated Teeth Based on Pain Status, Caries Color, and Hemostasis Status

\begin{tabular}{|l|l|l|l|}
\hline \multirow{5}{*}{} & & \multicolumn{2}{|l|}{ Hemostasis } \\
\cline { 3 - 4 } & & $\begin{array}{l}\text { Yes Number } \\
\text { of Teeth } \\
\text { (Percent in } \\
\text { the Row) }\end{array}$ & $\begin{array}{l}\text { No Number } \\
\text { of Teeth } \\
\text { (Percent in } \\
\text { the Row) }\end{array}$ \\
\hline Pain status & No pain & $\begin{array}{l}25(78.1 \%) \\
19(79.1 \%)\end{array}$ & $7(21.8 \%)$ \\
& Provoked pain & $(20.9 \%)$ \\
& Spontaneous pain & $5(50 \%)$ & $5(50 \%)$ \\
& Nocturnal pain & $5(35.7)$ & $9(64.3 \%)$ \\
\hline Caries color & Black & $26(78.7)$ & $7(21.2)$ \\
& Dark brown & $23(69.7)$ & $10(30.3)$ \\
& Light brown & $5(35.7)$ & $9(64.3)$ \\
& Yellow & $0(0 \%)$ & $0(0 \%)$ \\
\hline
\end{tabular}

caries. Hemostasis was achieved in most of the teeth with black dental caries (26 out of 33 teeth, $78.1 \%$ ), whereas in most of the teeth with light brown dental caries, hemostasis was not achieved ( 9 out of 14 teeth, 64.3\%).

The independent $t$-test showed that the mean ratio of the largest diameter of caries lesion to the mesiodistal width of the teeth (measured based on bitewing radiographs) in the teeth needing pulpectomy $(0.499 \pm 0.212)$ was statistically significantly higher than that of those needing pulpotomy $(0.404$ $\pm 0.151)(\mathrm{p}=0.024)$. Hemostasis was achieved in most of the teeth with distoocclusal caries (35 out of 44 teeth, 79.5\%), whereas in most of the teeth with mesioocclusal caries (17 out of 30 teeth, $56.6 \%$ ), hemostasis was not achieved (chi-square test, $\mathrm{p}=0.01$; Table 2).

\section{Discussion}

This study was performed to evaluate the relationship between the achievement of hemostasis and the variables

Table 2 The Frequency of the Treated Teeth Based on the Surface of the Tooth Involved with Caries and Hemostasis Status

\begin{tabular}{|c|c|c|c|}
\hline \multirow{2}{*}{$\begin{array}{l}\text { The Surface of } \\
\text { the Tooth } \\
\text { Involved with } \\
\text { Caries }\end{array}$} & \multicolumn{2}{|l|}{ Hemostasis } & \multirow[t]{2}{*}{ Total } \\
\hline & $\begin{array}{l}\text { Yes (Total =54) } \\
\text { Number of } \\
\text { the Teeth } \\
\text { (Percent in the } \\
\text { Row) }\end{array}$ & $\begin{array}{l}\text { No (Total =26) } \\
\text { Number of the } \\
\text { Teeth (Percent } \\
\text { in the Row) }\end{array}$ & \\
\hline Mesioocclusal caries & 13 (43.3\%) & 17 (56.6\%) & 30 (100\%) \\
\hline Occlusal caries & $6(100 \%)$ & $0(0.0 \%)$ & $6(100 \%)$ \\
\hline Distoocclusal caries & 35 (79.5\%) & 9 (20.4\%) & $44(100 \%)$ \\
\hline Total & $54(100 \%)$ & $26(100 \%)$ & $80(100 \%)$ \\
\hline
\end{tabular}

child pain status, caries color, and caries extent using bitewing radiographs at the canal orifice after preparing the access cavities. The results showed that hemostasis was achieved in most of the teeth with darker dental caries, whereas in most of the teeth with lighter dental caries, hemostasis was not achieved. Therefore, darker color caries probably indicated the treatability of the tooth with pulpotomy, whereas lighter color caries probably indicated the treatability of the tooth with pulpectomy. A previous study conducted by Bjørndal et al classified dental caries according to their colors into the three categories of light yellow (actively progressing caries), light brown (slowly progressing caries), and dark brown (slowly progressing/arrested caries). ${ }^{14}$ The relationship between carious lesion activity and the color of carious dentin before dentin removal and also the relationship between the caries color and the degree of caries progression in the lesion have been previously demonstrated. ${ }^{12} \mathrm{An}$ actively progressing carious dentin lesion often has a light yellow/beige color with a wet/moist surface texture which can be easily penetrated with a dental probe. In contrast, slowly progressing or arrested caries lesions are usually darker with no excess moisture and cannot be penetrated with a probe. ${ }^{14}$ The remnants of bacterial metabolism could destroy dentinal tissues which might be a possible cause of black or brown staining. ${ }^{17}$ The results of this study regarding caries color might be justified by the finding that active caries lesions have a lighter color compared to slowly progressing caries lesions or arrested caries lesions. ${ }^{14}$ However, visual inspection for evaluating caries color and carious lesion activity and progression is subjective and not numerical. ${ }^{12}$ Moreover, no yellow caries was detected in the teeth selected for this study. This finding might be related to the fact that the teeth with clear radiographic signs of pulpectomy were excluded from the study and might be more associated with yellow dental caries.

The current study also showed that the extent of dental caries in the teeth needing pulpotomy was statistically significantly lower than that of the teeth needing pulpectomy. In the current study, the teeth with extremely deep caries lesions in bitewing radiographs were selected. The most appropriate radiographic technique for caries detection is bitewing radiography which is an adjunct to clinical examination. Bitewing radiography can allow a more sensitive detection of occlusal and proximal caries lesions in dentin. Moreover, using bitewing radiography provides a better estimation of the lesion depth compared with 
visual inspection. ${ }^{15}$ This is the first study which correlates the extent of caries lesion measured in bitewing radiographs with the achievement of hemostasis. It was found that the mean ratio of the largest diameter of caries lesion to the mesiodistal width of the teeth (measured based on bitewing radiographs) needing pulpectomy was statistically significantly higher than that of those needing pulpotomy. It can be concluded that the extent of caries might be used as a potential diagnostic tool for predicting the treatability of the tooth with pulpotomy. This result is in line with a previous study which correlated the pulp pathology of primary teeth with the extent of the carious lesion as measured clinically in relation to the buccolingual intercuspal distance (ICD). This study concluded that where more than half of the ICD was involved with the carious process, more teeth demonstrated the evidence of inflammation extending to the pulp horn and the radicular pulp compared to where less than half of the ICD was involved with the carious process. ${ }^{10}$ Bitewing radiography was used to measure the extent of the caries lesion in the present study. However, it has some limitations. It has been demonstrated that the teeth with a proximal caries lesion extending more than $50 \%$ through the dentin thickness might have more extensive pulpal inflammation compared with those with an occlusal lesion of a similar depth. ${ }^{10}$ This can be used to interpret the findings of this study. Moreover, the results of this study revealed that hemostasis was achieved in most of the teeth with distoocclusal caries, whereas in most of the teeth with mesioocclusal caries, hemostasis was not achieved. No clear explanation was found for this finding. However, this finding might be due to the fact that the mesial pulp horns are higher and closer to the surfaces of the teeth compared to the distal ones. ${ }^{18}$ Therefore, the closer distance of the potential irritants to the pulp tissue on the mesial side compared to the distal side might affect the pulpal inflammation and achievement of hemostasis. Histologic studies are needed to find the exact explanation for the higher rate of hemostasis achievement in distoocclusal side caries compared to mesioocclusal ones.

Another criterion in this study related to the achievement of hemostasis at the canal orifice was preoperative pain status. According to the results of the present study, the frequency of the teeth that had no preoperative pain was statistically significantly more in cases which were treated with pulpotomy compared with those treated with pulpectomy. This finding showed that the lack of pain history in the teeth without any clear sign of pulpectomy treatment can be used as a predictive factor for the selection of pulpotomy. A previous study reported that spontaneous pain was more frequent in patients with severe pulp inflammation than those with mild or moderate pulpal inflammation. However, no statistically significant relationship was found between the different levels of pulp inflammation and provoked pain. ${ }^{5}$ A possible explanation for this finding is that activated pulpal leukocytes secrete some inflammatory mediators such as histamine, IL-1, and prostaglandins which stimulate the sensory nerves and cause pain. ${ }^{19}$ However, spontaneous or severe preoperative pain could not necessarily be indicative of an irreversible pattern of pulpal injury and the inability of the pulp to repair itself. ${ }^{20,21}$ Nevertheless, it is difficult to assess pain in children because an accurate description of the history of pain might not be obtained in children. ${ }^{22,23}$ The results of the current study showed that most of the teeth with nocturnal pain (64.3) needed pulpectomy. This finding is in agreement with the results of many studies which have demonstrated that nocturnal pain is related to irreversible pulpitis. ${ }^{24-26}$ For the same reason mentioned about the frequency of dental caries color, only $17.5 \%$ of the teeth had nocturnal pain as the teeth with a clear radiographic sign of pulpectomy were excluded from the study. The existence of nocturnal pain is more likely in them compared with those without a clear radiographic sign of pulpectomy. However, $35.7 \%$ of the teeth with nocturnal pain showed hemostasis after the preparation of access cavities. This finding might be due to food impaction which could result in nocturnal pain.

This study has some limitations. Although caries color was used in the present study to assess the caries activity in clinical practice, hardness is a more reliable indicator for the assessment of caries activity which should be considered in future studies. ${ }^{27}$ Long-term prospective randomized data are necessary to assess the results of the selected treatments and to verify the findings of the current study. In the current study, caries color, caries extent, and preoperative pain status correlated with the achievement of hemostasis. However, these potential diagnostic methods might only provide clinical guidance and have such limitations as being subjective and relatively crude. Additionally, they are not able to reflect the true histopathological status of the pulp. ${ }^{9}$ Moreover, it would be beneficial to use standard light sources, charged-coupled device (CCD) video cameras, automatic computer programs, and colorimeter devices in future studies for the evaluation of caries color. 


\section{Conclusion}

Within the limitations of the present study, it can be concluded that the caries color, caries extent, and preoperative pain have the potential to be used as valid clinical diagnostic criteria of pulpal status for the selection of an appropriate pulp treatment strategy.

\section{Acknowledgments}

The authors thank the Vice-Chancellery of Research, Shiraz University of Medical Sciences for supporting this research (\#97-01-03-19239). This article is based on Dr. Alireza Tadayon's thesis. The authors thank Dr. Mehrab Sayadi from the Dental Research Development Center for the statistical analyses. The authors also thank Dr. Arash Moradi for editorial assistance.

\section{Disclosure}

The authors report no conflicts of interest in this work.

\section{References}

1. Fuks AB. Vital pulp therapy with new materials for primary teeth: new directions and treatment perspectives. Pediatr Dent. 2008;30(3):211-219.

2. Shantiaee Y, Javaheri S, Movahhedian A, Eslami S, Dianat O. Efficacy of preoperative ibuprofen and meloxicam on the success rate of inferior alveolar nerve block for teeth with irreversible pulpitis. Int Dent J. 2017;67(2):85-90. doi:10.1111/idj.12272

3. Ghaderi F, Ghaderi R, Davarmanesh M, Bayani M, Moghadam SA. Pain management during needle insertion with low level laser. Eur J Paediatr Dent. 2016;17:151-154

4. Gopinath VK, Anwar K. Histological evaluation of pulp tissue from second primary molars correlated with clinical and radiographic caries findings. Dent Res J. 2014;11(2):199-203.

5. Aaminabadi NA, Parto M, Emamverdizadeh P, Jamali Z, Shirazi S. Pulp bleeding color is an indicator of clinical and histohematologic status of primary teeth. Clin Oral Investig. 2017;21(5):1831-1841. doi:10.1007/s00784-017-2098-y

6. Chen E, Abbott PV. Dental pulp testing: a review. Int $J$ Dent. 2009;2009:1-12. doi:10.1155/2009/365785

7. Mutluay M, Arıkan V, Sarı S, Kısa Ü. Does achievement of hemostasis after pulp exposure provide an accurate assessment of pulp inflammation? Pediatr Dent. 2018;40(1):37-42.

8. Hori A, Poureslami HR, Parirokh M, Mirzazadeh A, Abbott P. The ability of pulp sensibility tests to evaluate the pulp status in primary teeth. Int J Paediatr Dent. 2011;21(6):441-445. doi:10.1111/j.1365263X.2011.01147.x

9. Duncan H, Galler K, Tomson P, et al. European Society of Endodontology position statement: management of deep caries and the exposed pulp. Int Endod J. 2019;52(7):923-934. doi:10.1111/iej.13080

10. Kassa D, Day P, High A, Duggal M. Histological comparison of pulpal inflammation in primary teeth with occlusal or proximal caries. Int J Paediatr Dent. 2009;19(1):26-33. doi:10.1111/j.1365263X.2008.00962.x
11. Bjørndal L, Simon S, Tomson P, Duncan H. Management of deep caries and the exposed pulp. Int Endod J. 2019;52(7):949-973. doi:10.1111/iej.13128

12. Iwami Y, Hayashi N, Takeshige F, Ebisu S. Relationship between the color of carious dentin with varying lesion activity, and bacterial detection. J Dent. 2008;36(2):143-151. doi:10.1016/j.jdent.2007.11.012

13. Banerjee A, Watson T, Kidd E. Dentine caries: take it or leave it? Dent Update. 2000;27(6):272-276. doi:10.12968/denu.2000.27.6.272

14. Bjørndal L, Larsen T, Thylstrup A. A clinical and microbiological study of deep carious lesions during stepwise excavation using long treatment intervals. Caries Res. 1997;31(6):411-417. doi:10.1159/ 000262431

15. Wenzel A. Bitewing and digital bitewing radiography for detection of caries lesions. J Dent Res. 2004;83(1_suppl):72-75. doi:10.1177/ $154405910408301 \mathrm{~s} 14$

16. Arnold WH, Bietau V, Renner PO, Gaengler P. Micromorphological and microanalytical characterization of stagnating and progressing root caries lesions. Arch Oral Biol. 2007;52(6):591-597. doi:10.1016/j.archoralbio.2006.11.008

17. Kuboki Y, Liu C-F, Fusayama T. Mechanism of differential staining in carious dentin. J Dent Res. 1983;62(6):713-714. doi:10.1177/ 00220345830620060401

18. Duggal M, Nooh A, High A. Response of the primary pulp to inflammation: a review of the leeds studies and challenges for the future. Eur J Paediatr Dent. 2002;3(3):111-114.

19. Yadlapati M, Biguetti C, Cavalla F, et al. Characterization of a vascular endothelial growth factor-loaded bioresorbable delivery system for pulp regeneration. $J$ Endod. 2017;43(1):77-83. doi:10.1016/j.joen.2016.09.022

20. Ricucci D, Loghin S, Siqueira JJF. Correlation between clinical and histologic pulp diagnoses. $J$ Endod. 2014;40(12):1932-1939. doi:10.1016/j.joen.2014.08.010

21. Taha NA, Abdulkhader SZ. Full pulpotomy with biodentine in symptomatic young permanent teeth with carious exposure. J Endod. 2018;44(6):932-937. doi:10.1016/j.joen.2018.03.003

22. Wong M, Copp PE, Haas DA. Postoperative pain in children after dentistry under general anesthesia. Anesth Prog. 2015;62(4):140-152. doi:10.2344/14-27.1

23. El Batawi H. Effect of intraoperative analgesia on children's pain perception during recovery after painful dental procedures performed under general anaesthesia. Eur J Paediatr Dent. 2015;16(1):35-41. doi:10.1007/s40368-014-0143-y

24. Sooraparaju SG, Abarajithan M, Sathish ES, Suryakumari NBP, Ealla KKR, Gade W. Anaesthetic efficacy of topical benzocaine gel combined with hyaluronidase for supplemental intrapulpal injection in teeth with irreversible pulpitis a double blinded clinical trial. J Clin Diagn Res. 2015;9(8):ZC95-ZC97. doi:10.7860/JCDR/2015/12575.6401

25. Mehrvarzfar P, Pourhashemi A, Khodaei F, et al. The effect of adding fentanyl to epinephrine-containing lidocaine on the anesthesia of maxillary teeth with irreversible pulpitis: a randomized clinical trial. Iran Endod J. 2014;9(4):290-294.

26. Alyahya A, Khanum A, Qudeimat M. Clinical assessment of class II resin-based composites versus preformed metal crowns performed on primary molars in patients at high risk of caries. Eur J Paediatr Dent. 2018;19(1):39-45. doi:10.1007/s40368-017-0322-8

27. Hon L, Mohamed A, Lynch E. Reliability of colour and hardness clinical examinations in detecting dentine caries severity: a systematic review and meta-analysis. Sci Rep. 2019;9(1):1-11. doi:10.1038/s41598-019-41270-6 


\section{Publish your work in this journal}

Clinical, Cosmetic and Investigational Dentistry is an international, peer-reviewed, open access, online journal focusing on the latest clinical and experimental research in dentistry with specific emphasis on cosmetic interventions. Innovative developments in dental materials, techniques and devices that improve outcomes and patient satisfaction and preference will be highlighted. The manuscript management system is completely online and includes a very quick and fair peer-review system, which is all easy to use. Visit http://www.dovepress.com/testimonials.php to read real quotes from published authors. 